\title{
三维打印羟基磷灰石晶须增强复合骨修复支架
}

\author{
辛晨 ${ }^{1}$, 齐鍂 ${ }^{2}$, 朱 敏 ${ }^{1}$, 赵世昌 ${ }^{2}$, 朱钰方 ${ }^{1}$
}

(1. 上海理工大学 材料科学与工程学院, 上海 200093；2. 上海交通大学附属第六人民医院 骨外科, 上海 200233)

摘 要: 利用三维打印技术成功制备羟基磷灰石晶须(HAPw)增强的聚己内酯(PCL)复合骨修复支架。通过改变三维 打印的挤出速度和挤出气压, 使不同含量 HAPw 均能在 PCL 基材中一致排列并均匀分布。PCL 支架的机械强度随 $\mathrm{HAPw}$ 含量增加显著提高, 添加 $33 \mathrm{wt} \% \mathrm{HAPw}$ 使 PCL 支架强度提升了高达 3 倍。此外, HAPw 使 PCL 支架表面与 水的接触角从近 $100^{\circ}$ 降低至约 $50^{\circ}$, 有效改善了细胞表面粘附。经过体外人类骨髓间充质干细胞(hBMSC)在支架上 的培养实验, 发现添加 HAPw 的复合支架具有更好的生物相容性, 能够有效促进 hBMSC 的增殖生长, 且 HAPw-PCL 复合支架上细胞具有更高的碱性磷酸酶(ALP)活性和 OCN、RUNX2 等相关成骨基因表达，显示出 hBMSCs 向成骨方向更好的分化及成骨活性。

关 键 词: 三维打印; 羟基磷灰石晶须; 支架; 骨修复

中图分类号: TQ174 文献标识码: A

\section{Hydroxyapatite Whisker-reinforced Composite Scaffolds Through 3D Printing for Bone Repair}

\author{
XIN Chen ${ }^{1}$, QI Xin ${ }^{2}$, ZHU Min ${ }^{1}$, ZHAO Shi-Chang ${ }^{2}$, ZHU Yu-Fang ${ }^{1}$ \\ (1. School of Materials Science and Engineering, University of Shanghai for Science and Technology, Shanghai 200093, China; \\ 2. Department of Orthopedic Surgery, Shanghai Sixth People's Hospital, Shanghai Jiaotong University, Shanghai 200233, China)
}

\begin{abstract}
Development of bioactive scaffolds with controllable architecture and high osteogenic capability is essential for bone tissue engineering. In this study, hydroxyapatite whiskers (HAPw) were added into polycaprolactone (PCL) matrix materials to fabricate scaffolds through 3D printing technique. The HAPw could distribute homogeneously in PCL and in alignment with 3D printing directions by adjusting squeeze parameters. The mechanical strength of PCL-HAPw composite scaffolds increased along with the increase of HAPw content. Adding $33 \mathrm{wt} \%$ of HAPw remarkably enhanced the compressive strength of PCL scaffolds to 3 times, which can also lower the surface contact angles from $100^{\circ}$ of PCL to $50^{\circ}$ and thus enhance the surface hydrophilicity. In vitro culturing experiments of human bone marrow mesenchymal cells (hBMSCs) demonstrated that the incorporation of HAPw promoted their bioactive and osteogenic properties, including better cytocompatibility, cell adhesion, proliferation, alkaline phosphatase (ALP) activity, and bone-related gene expressions (OCN, RUNX2). Therefore, 3D-printed HAPw-PCL composite scaffolds showed improved mechanical strength and osteogenesis properties compared to pure PCL scaffolds, and suggest promising applications in bone regeneration.
\end{abstract}

Key words: 3D printing; hydroxyapatite whiskers; scaffolds; bone regeneration

收稿日期: 2016-11-15; 收到修改稿日期：2016-12-21

基金项目：国家自然科学基金青年项目(51302170)；上海市教委科研创新项目(14YZ085)

National Natural Science Foundation of China (51302170); Innovation Program of Shanghai Municipal Education Commission (14YZ085)

作者简介：辛晨(1990-), 男, 硕士研究生. E-mail: xinchen517@163.com

通讯作者: 朱 敏, 副教授. E-mail: mzhu@usst.edu.cn 
近年来运用组织工程技术修复骨缺损是生物材 料研究领域的热点之一 ${ }^{[1]}$ 。骨组织工程使用的三维 多孔支架作为细胞载体支持成骨相关细胞生长及营 养物质和代谢产物的运输, 对骨修复效果起着决定 性作用。

从组成上来说, 具有良好相容性的可降解生物高 分子是一类常用的支架材料, 例如, 聚己内酯 $[2-3]$ 聚乳

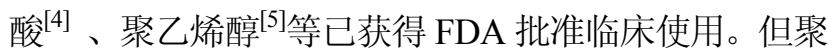
合物存在机械强度差，降解产物普遍呈酸性等缺点， 因而通过添加无机组份模拟天然骨组织组成, 增强聚 合物支架强度，同时改善其生物活性，具有实际意义。

羟基磷灰石 $\left(\mathrm{HAP}, \mathrm{Ca}_{10}\left(\mathrm{PO}_{4}\right)_{6}(\mathrm{OH})_{2}\right)$ 类似于人类 骨骼的主要无机成分, 具有良好的骨传导性和生物 相容性 ${ }^{[6-7]}$, 可与宿主骨组织形成直接的骨性结合, 是良好的硬组织替代材料。目前已可控合成了多种 形貌和尺寸的 HAP 材料, 用于直接制备骨修复植入 体或作为有机支架的增强相材料。经研究表明, 不 同形貌的增强相 HAP 因表现出不同的增强效果, 其 中 HAP 晶须(HAPw)的增强效果明显优于 HAP 球形 纳米颗粒、棒或片 ${ }^{[8-13]}$ 。HAPw 加入聚合物基体中 的含量、分散程度和排列取向与最终增强效果密切 相关。传统骨修复有机/无机复合支架制备方法，如 溶液浇铸/粒子沥滤法 ${ }^{[14]}$ 、相分离法/冷冻干燥法 ${ }^{[15]}$ 和有机泡沫浸渍法/模板法 ${ }^{[16]}$ 等, 基本依靠机械混 合的方法复合无机增强相和有机高聚物, 导致晶须 分散度低, 分布浓度不均匀, 甚至可能出现显著的 分相行为或大面积有机无机界面, 削弱了晶须的增 强效果。因此，寻找合适技术手段制备晶须增强复 合支架是有必要的。气动挤压浆料成型技术在生物 3D 打印的应用上具有优势, 可选材料范围广泛、细 胞能与材料同时成形、支架孔隙结构可精确控制。 含晶须材料的聚合物基体经由气动挤出后再三维打 印成型, 将使支架的均匀度得到大大提升, 获得平 行于打印方向的一致取向, 进而提高支架的力学强 度。另外, 这种三维打印技术跟临床计算机断层扫 描、磁共振成像等数据结合, 可以通过重建模型直 接快速制造任意复杂形状的三维植入体，完成个人 医疗产品的定制, 在个性化精准医疗领域具有强大 的应用潜力 ${ }^{[17-19]}$ 。

本研究利用气动挤压三维打印技术, 将预先制 备好的 HAPw 与聚己内酯溶液共混, 以 PCL 为主体 材料, HAPw 为增强体, 制备出不同比例的 HAPwPCL 复合支架。考察不同比例复合支架的微观结 构、亲疏水特性和力学性能等, 并在支架上培养人 骨髓间充质干细胞(hBMSCs), 检测其粘附、增殖和
分化行为, 以期实现具有良好成骨活性、结构可控 和合适机械强度的 HAPw 增强复合骨修复支架。

\section{1 材料和方法}

\section{1 试剂与原料}

硝酸 $\left(\mathrm{HNO}_{3}, 65 \%\right)$, 氯仿 $\left(\mathrm{CHCl}_{3}\right)$ ，二甲基亚 砜 (DMSO), 磷酸氢二铵 $\left(\left(\mathrm{NH}_{4}\right)_{2} \mathrm{HPO}_{4}, 99 \%\right)$, 四 水硝酸钙 $\left(\mathrm{Ca}\left(\mathrm{NO}_{3}\right)_{2} \cdot 4 \mathrm{H}_{2} \mathrm{O}, 99 \%\right)$ 和乙酰胺 $\left(\mathrm{CH}_{3} \mathrm{CO}\right.$ $\mathrm{NH}_{2}, 98.5 \%$ ), 纳米 HAP 粉体(HAPnp)均购于国药集 团化学试剂有限公司。聚己内酯 (PCL, 分子量 70000 90000)购于 Sigma-Aldrich。

\section{$1.2 \mathrm{HAPw}$ 的水热制备}

将 $4.955 \mathrm{~g} \mathrm{Ca}\left(\mathrm{NO}_{3}\right)_{2} \cdot 4 \mathrm{H}_{2} \mathrm{O} 、 1.65 \mathrm{~g}\left(\mathrm{NH}_{4}\right)_{2} \mathrm{HPO}_{4}$ 和 $27 \mathrm{~g} \mathrm{CH}_{3} \mathrm{CONH}_{2}$ 依次加入到 $500 \mathrm{~mL} 0.05 \mathrm{~mol} / \mathrm{L}$ 的 $\mathrm{HNO}_{3}$ 中，摚拌 $5 \mathrm{~h}$ 。待完全溶解后，将混合溶液 转移至水热釜中, 在 $180^{\circ} \mathrm{C}$ 反应 $10 \mathrm{~h}$, 产物抽滤分离, 超纯水洗涤，干燥待用。

\section{3 三维打印制备 HAPw-PCL 复合支架}

使用第四代 3D Bioplotter ${ }^{\mathrm{TM}}$ (Envision TEC $\mathrm{GmbH}$, Germany)三维打印机制备 HAPw-PCL 复合 支架。首先按照表 1 配制不同组成的打印浆料, 然 后气动挤压浆料逐层堆积制备三维支架。打印参数: 气体压力 $(1.0 \sim 4.0) \times 10^{5} \mathrm{~Pa}$, 打印速度 $5 \sim 10 \mathrm{~mm} / \mathrm{s}$, 针头尺寸 $0.4 \mathrm{~mm}$ 。将 $\mathrm{HAPw}-\mathrm{PCL}$ 支架置于 $37^{\circ} \mathrm{C}$ 烘 箱中干燥两天。并按照相同流程制备参照用纯 PCL 支架和 HAPnp 复合支架。样品命名见表 1 。

\subsection{HAPw-PCL 复合支架的形貌、抗压强} 度、孔隙率以及亲水性测试

在 Bruker D8 Advance 上测试样品的广角 XRD 图谱; 在 FEI Quanta 450 型场发射扫描电镜上观察 HAPw 和 HAPw-PCL 支架的形貌。利用 $2.5 \mathrm{kN}$ 的 Zwick 静态材料试验机测试支架的机械强度，测试

表 1 复合支架编号及打印浆料组成

Table1 Composite scaffold number and composition of printing paste

\begin{tabular}{cccccc}
\hline Samples & $\begin{array}{c}\mathrm{HAPw} \\
/ \mathrm{g}\end{array}$ & $\begin{array}{c}\mathrm{HAPnp} \\
/ \mathrm{g}\end{array}$ & $\begin{array}{c}\mathrm{PCL} \\
/ \mathrm{g}\end{array}$ & $\begin{array}{c}\mathrm{CHCl}_{3} \\
/ \mathrm{mL}\end{array}$ & $\begin{array}{c}\mathrm{DMSO} \\
/ \mathrm{mL}\end{array}$ \\
\hline HAPnp-2PCL & 0 & 0.5 & 1 & 3.8 & 0.2 \\
HAPw-2PCL & 0.5 & 0 & 1 & 3.8 & 0.2 \\
HAPw-5PCL & 0.2 & 0 & 1 & 3.8 & 0.2 \\
HAPw-10PCL & 0.1 & 0 & 1 & 3.8 & 0.2 \\
PCL & 0 & 0 & 1 & 3.8 & 0.2 \\
\hline
\end{tabular}


速度为 $0.5 \mathrm{~mm} / \mathrm{min}$, 支架的尺寸为 $10 \mathrm{~mm} \times 10 \mathrm{~mm} \times$ $5 \mathrm{~mm}$ 。运用阿基米德方法测量支架的孔隙率, 测试 介质为超纯水, 孔隙率计算公式如下:

$$
P=\left(W_{\text {sat }}-W_{\text {dry }}\right) /\left(W_{\text {sat }}-W_{\text {sus }}\right) \times 100 \%
$$

其中 $W_{\text {dry }}$ 为支架完全干燥状况下的重量, $W_{\text {sus }}$ 为支 架悬浮在水中的重量, $W_{\text {sat }}$ 为支架从水中取出时的 重量。

使用打印制备的无孔隙不同比例支架单层薄片 样品(10 mm $\times 10 \mathrm{~mm})$ 测试样品的亲水性, 用 CA100D 静/动态接触角测试软件计算接触角大小。

\section{5 人体骨髓间充质干细胞(hBMSCs)的分离} 与培养

根据文献[20]方法分离 hBMSCs, 细胞实验过 程中使用到的人体样本得到了上海交通大学附属第 六人民医院的批准。实验所用为第四 七代细胞。

\section{6 hBMSCs 在 HAPw-PCL 复合支架上的增殖}

通过 CCK-8 试剂盒进行细胞计数, 检测 hBM$\mathrm{SCs}$ 在各支架上的生长增殖情况。步骤如下: 以 $1 \times 10^{4}$ 密度在每个支架上种植细胞, 共同培养 3 和 $7 \mathrm{~d}$ 后, 每孔分别加入 $360 \mu \mathrm{L}$ 培养基和 $40 \mu \mathrm{L} \mathrm{CCK}-8$ 溶液, 在 $37^{\circ} \mathrm{C}, 5 \% \mathrm{CO}_{2}$ 气氛的培养箱中培养 $4 \mathrm{~h}$, 各 孔取出 $100 \mu \mathrm{L}$ 溶液转移到新 96 孔板上, 通过酶标 仪(BioRad 680, USA)测试在 $450 \mathrm{~nm}$ 处的吸光度。

\section{7 hBMSCs 在 HAPw-PCL 复合支架上的成 骨分化}

通过检查 hBMSCs 在各支架上的碱性磷酸酶 (ALP)活性和相关成骨基因表达(ALP、RUNX2、

OCN), 测试 hBMSCs 向成骨方向分化能力。

将 $1 \times 10^{5}$ 密度的细胞种植到每组支架上, 在 24 孔板上共培养 7 和 $14 \mathrm{~d}$; 除去培养基, 用磷酸盐缓冲 液冲洗细胞层 3 次, 再用 $50 \mathrm{mmol} / \mathrm{L}$ 的 Tris 缓冲溶液 清洗，用 $200 \mu \mathrm{L} 0.2 \%$ Triton X-100 溶液将细胞溶解, 超声分散, 离心分离, 取上层 $50 \mu \mathrm{L}$ 清液和 $150 \mu \mathrm{L}$ 标 准试剂(Beyotime)混合, 在酶标仪(Bio-Rad 680, USA) 的 $405 \mathrm{~nm}$ 处读取 OD 值。同时按照 BCA 法蛋白定 量检测试剂盒的说明, 进行蛋白定量检测, 以 $\mu \mathrm{mol} /(\mathrm{L} \cdot \mathrm{min} \cdot \mathrm{mg})$ protein 表示 ALP 活性。

采用 qRT-PCR 技术检测成骨基因 ALP、Runt 相关转录因子 (RUNX2)、骨钙蛋白 $(\mathrm{OCN})$ 的表达。 首先将 $2 \times 10^{4}$ 密度的细胞种植到每组支架上培养两 周, 运用 TRIzol Reagent (Invitrogen Pty Ltd, Australia)提取 RNA, 运用 RevertAid First Strand cDNA Synthesis Kit (Thermo)反转录 DNA(cDNA), 运用 SYBR Green 检测试剂进行 qRT-PCR(ABI Prism 7300 Thermal Cycler(Applied Biosystems, Australia))
分析，三组平行试验并独立取平均值 $(C \mathrm{t})$ 。基因表达 计算公式如下:

$$
P=2^{-(\text {normalized average } C \mathrm{t})} \times 100
$$

1.8 hBMSCs 在 HAPw-PCL 复合支架上细胞 外基质矿化研究

hBMSCs 在支架上培养 $21 \mathrm{~d}$ 后，用 PBS 缓冲液 清洗三遍，并用 $4 \%(W / V)$ 多聚甲醛(PFA)溶液在室温 下固定 $15 \mathrm{~min}$, 用 $2 \%(W / V)$ 的茜素红溶液在 $\mathrm{pH}$ 为 4.2 下保持 $15 \mathrm{~min}$, 最后用超纯水把细胞清洗下来, 在相衬显微镜下观察。

\section{2 结果与讨论}

\subsection{HAPw 形貌}

如图 1 所示, 水热合成的 HAPw 呈现较为均匀 的尺寸分布和良好的分散性，晶须直径约为 100 $400 \mathrm{~nm}$, 晶须长度大致为 60 200 $\mu \mathrm{m}$, 因此长径比 可高达 500。

\subsection{HAPw-PCL 复合支架的形貌、抗压能} 力、孔隙率以及亲水性

图 2 为 HAPw、纯 PCL 和 HAPw-PCL 复合支 架的 XRD 图谱,如图所示, HAPw 的衍射峰与羟基磷 灰石标准图谱(JCPDS 54-0022)一致。另外, HAPw-
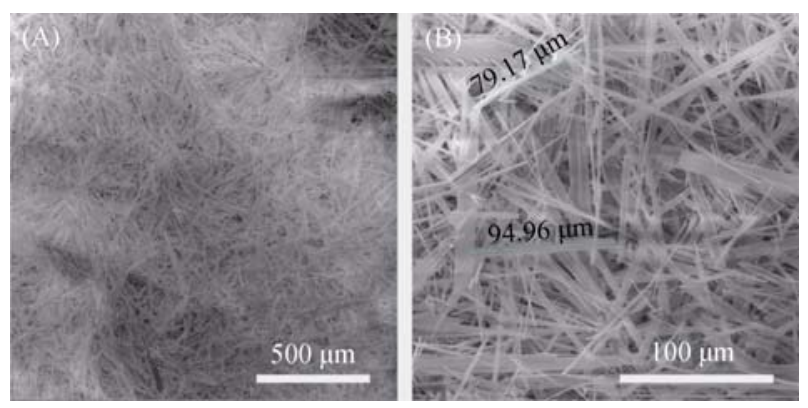

图 $1 \mathrm{HAPw}$ 的不同放大倍数下扫描电镜照片

Fig. 1 SEM images of HAPw at different magnifications

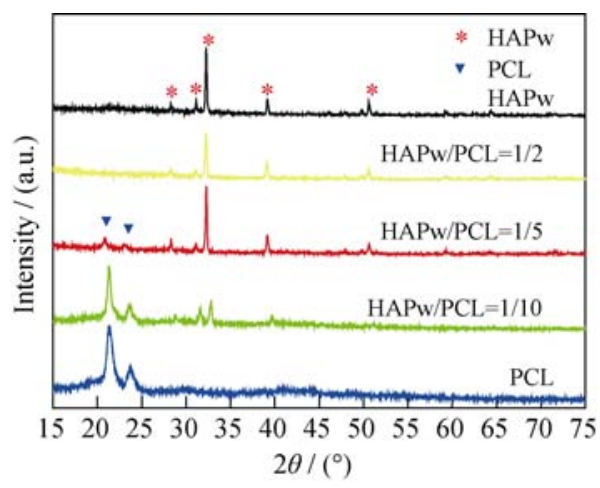

图 2 HAPw、PCL 和 HAPw-PCL 支架的 XRD 图谱

Fig. 2 XRD patterns of HAPw, PCL and HAPw-PCL scaffolds 
10PCL、HAPw-5PCL 和 HAPw-2PCL 复合支架的衍 射峰包含 HAPw 和 PCL 特征峰, 表明三维打印过程 对复合支架组成的 HAPw 和 PCL 材料末产生影响。 随着复合支架中 $\mathrm{HAPw}$ 含量的逐步增加, HAPw 衍 射峰的强度明显上升。

纯 PCL、HAPnp-2PCL 和各种组成比例的 HAPw-PCL 复合支架的 SEM 照片如图 3 所示。可 以看到, 所有支架均具有 X-Y 交叉堆积的孔隙结构, 由于 HAPw 或 HAPnp 无机组成比例的不同, 造成打 印浆料黏度和可打印性的明显差别, 因而获得的支 架最终孔道尺寸与打印预设尺寸存在差异。例如, 图 3 中纯 PCL 支架明显坞塌, 导致打印挤出支宽度 接近 $800 \mu \mathrm{m}$; 而 HAPw-5PCL 支架除上下层打印支 接触处有稍微宽化以外, 支架孔洞保持在 $400 \mu \mathrm{m}$ 左右。放大照片显示支架表面的微观形貌与结构。

HAPw/PCL 系列复合支架均可观察到表面覆盖有 PCL 黏结剂, 而且由于氯仿溶剂在打印过程中的快 速挥发和 HAPw 的支撑, 造成 PCL 覆盖层上出现大 量次级孔隙, 且这种孔随着 HAPw 含量增加相应增 大, 如 HAPw-2PCL 支架样品, 在表面可清楚地观 察到晶须轮廓, PCL 未分相单独形成包裹支架的薄 膜层。对于 HAPnp 纳米颗粒与 PCL 的复合支架, 表 面致密度要远高于 HAPw-PCL 支架, 这可能是由于 纳米颗粒的密堆程度更高。这种在表面形成的 PCL 膜亲水性能可能较差, 将直接阻碍细胞与支架中其 它组份尤其是 HAP 组份的接触和相互作用, 不利于 细胞的粘附和增殖。值得注意的是, HAPw 材料在复 合支架中的排列呈现有序, 并与打印方向一致, 从 图 3 中可以看出, 晶须方向统一且在基底中分散均
匀, 大大改善了传统机械混合工艺带来的有机无机 微区分相问题。

由阿基米德方法测试计算纯 PCL、HAPw10PCL、HAPw-5PCL、HAPw-2PCL 和 HAPnp-2PCL 五组支架的孔隙率分别为 $(41.71 \pm 2.41) \%$ 、 $(67.26 \pm$ $1.13) \%$ 、(69.10 1.96$) \%$ 、(71.37 \pm 1.85$) \%$ 和 (59.52 2.25 )\% (图 4), 其相对大小与 SEM 表征结果基本吻合。 根据打印参数计算, 直径 $400 \mu \mathrm{m}$ 、支间间隙 $0.8 \mathrm{~mm}$ 的 支架理论孔隙率应为 $76.25 \%$, 实际孔隙率则有一定 偏差。如纯 PCL 支架中打印支的妇塌导致孔隙率偏 低, 而孔道最为规则的 HAPw-5PCL 支架则与理论 值接近。不同组成比例的 HAPw-PCL 复合支架孔隙 率并无明显差异。研究表明, 理想情况下, 高孔隙度 且具有联通孔隙的支架有利于骨再生, 并能促进细 胞增殖、血管生成和内部矿化骨形成。三维打印技 术则可以简便地改变打印参数，包括支架各打印支 的距离、尺寸和层间角度，获得大范围可调的孔隙 结构，并对于不同组成的材料打印也可进行调节， 尽量获得符合要求的支架结构。

图 5 列出了各组支架样品的抗压强度测试结果, 五组支架的抗压强度分别为 $(3.14 \pm 0.36) 、(4.07 \pm$ $0.42) 、(5.80 \pm 0.93) 、(9.75 \pm 1.09)$ 和 $(8.58 \pm 1.04) \mathrm{MPa}$, 均在人体松质骨强度值范围内 $(2 \sim 12 \mathrm{MPa})$ 。添加 HAPw 和 HAPnp 的复合支架与纯 PCL 支架相比，强 度明显提高。其中, 随着 HAPw 含量增加, 抗压强 度越高。HAPw-PCL 复合支架的强度较高主要得益 于以下三个方面: 1)具有高长径比的 HAP 晶须穿插 在 PCL 基体中且分散均匀，有效缓解了外部冲击和 应力；2)三维打印得到的支架孔道整齐有序，尺寸
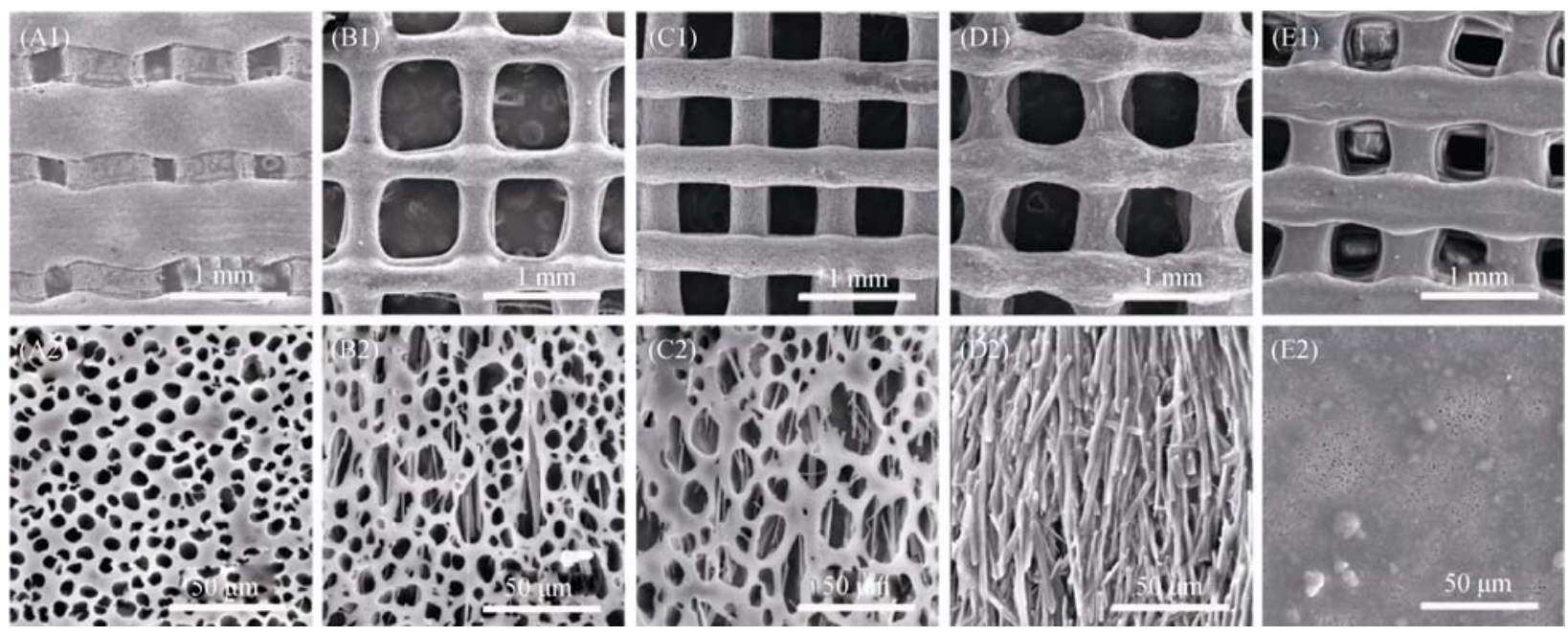

图 3 三维打印的 PCL(A1, A2), HAPw-10PCL(B1, B2), HAPw-5PCL(C1, C2), HAPw-2PCL(D1, D2)和 HAPnp-2PCL 支架的 SEM 照片

Fig. 3 SEM images of 3D-printed PCL(A1,A2), HAPw-10PCL (B1,B2), HAPw-5PCL (C1,C2), HAPw-2PCL (D1,D2) and HAPnp-2PCL scaffolds 


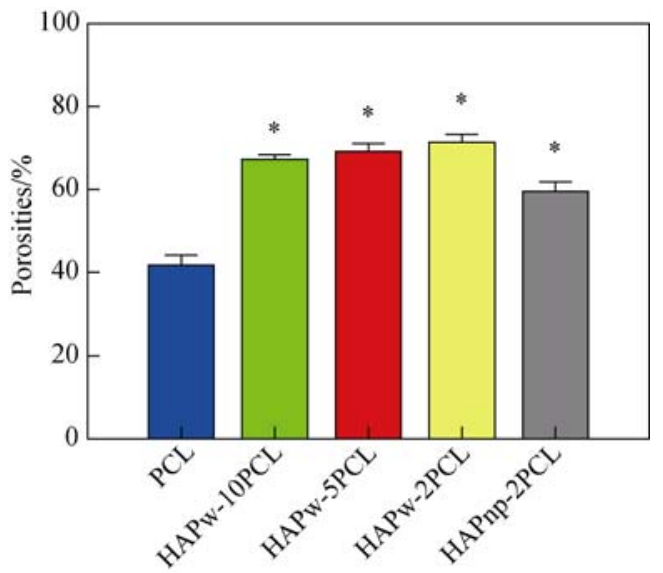

图 4 PCL、HAPnp-PCL 和 HAPw-PCL 支架的孔隙率测试结果 Fig. 4 Porosity of PLC, HAPw-PCL and HAPnp-PCL scaffold $\left({ }^{*} p<0.05\right.$ if compared with the PCL scaffold control)

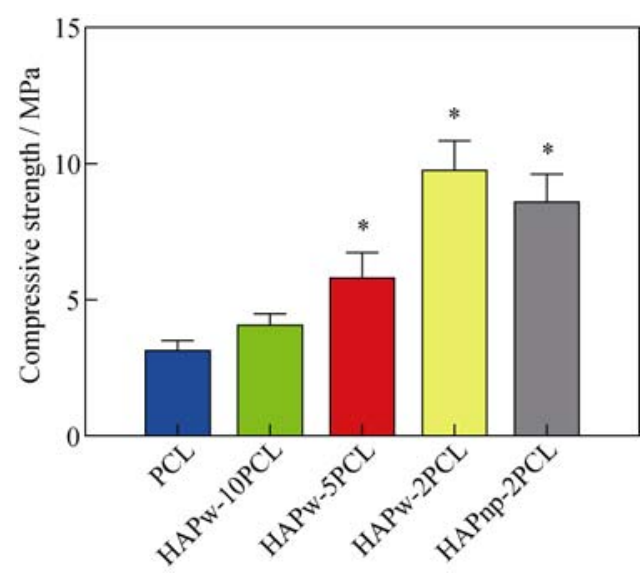

图 5 PCL、HAPnp-PCL 和 HAPw-PCL 支架的抗压能力测试结果 Fig. 5 Compressive strength of the PCL, HAPw-PCL and HAPnp-PCL scaffold $\left({ }^{*} p<0.05\right.$ if compared with the PCL scaffold control)
相近，各打印支间连接牢固，支架整体体现出较高 的均一性，有利于提高结构上承受压力的能力；3) 在复合材料中，载荷主要由基体承受。将 HAP 晶须 分散在基体中, 它可以阻碍基体中的位错运动或分 子链的运动。本研究中羟基磷灰石晶须以阻碍聚己 内酯分子链运动的方式达到了增强聚已内酯的效 果。另外，具有相同比例 HAP 的 HAPw-2PCL 支架 和 HAPnp-2PCL 支架, 纳米颗粒增强效果比纳米晶 须的差，这与已报道结果相吻合。例如，Assa 等 ${ }^{[21]}$ 将共混法与溶剂挥发法相结合，在左旋聚乳酸 (PLLA) 中添加 0 50vol\% 纳米羟基磷灰石颗粒 (HAPnp)，抗压屈服强度由 $50 \mathrm{MPa}$ 提高到 $96 \mathrm{MPa}$, 杨氏模量由 $2 \mathrm{GPa}$ 提高至 $6 \mathrm{GPa}$ 。Hayati 等 ${ }^{[22]}$ 利用 压缩模塑技术，在 PHB 支架中加入 $0 \sim 20 \mathrm{wt} \%$ 的 HAPnp, 当 HAPnp 含量为 $15 \mathrm{wt} \%$ 时, 性能达到最佳, 抗压强度由 $2.03 \mathrm{MPa}$ 提高到 $3.19 \mathrm{MPa}$, 压缩模量由 $29.06 \mathrm{MPa}$ 提高到 $59.12 \mathrm{MPa}$, 而当 HAPnp 含量达 到 $20 \mathrm{wt} \%$ 时，由于颗粒团聚导致抗压强度和压缩模 量均有所降低。Zhang 等 ${ }^{[23]}$ 利用相分离法, 在左旋聚乳 酸(PLLA)中添加 50vol\%HAPnp 可获得与纯 PLLA 支架 相比, 增强约 $30 \%$ 的抗拉模量和屈服强度。而抗压强度 测试结果表明, HAPw-2PCL 具有最好的增强效果, 抗 压强度由(3.14 \pm 0.36$) \mathrm{MPa}$ 提高至(9.75 \pm 1.09$) \mathrm{MPa}$, 增强效果提升约 3 倍, 明显优于 HAPnp 的增强效果。

图 6 为各组成无大孔支架表面亲水性能测试结 果, 水滴在表面的润湿接触角随着 HAPw 含量增加 而有所下降。由图 6 可以看出, 纯 PCL 支架在接触 测试 $20 \mathrm{~min}$ 后仍具有近 $100^{\circ}$ 接触角, 而 $\mathrm{HAPw}-$ $2 \mathrm{PCL}$ 复合支架的接触角小于 $50^{\circ}$, 表明添加 $\mathrm{HAPw}$
(A)
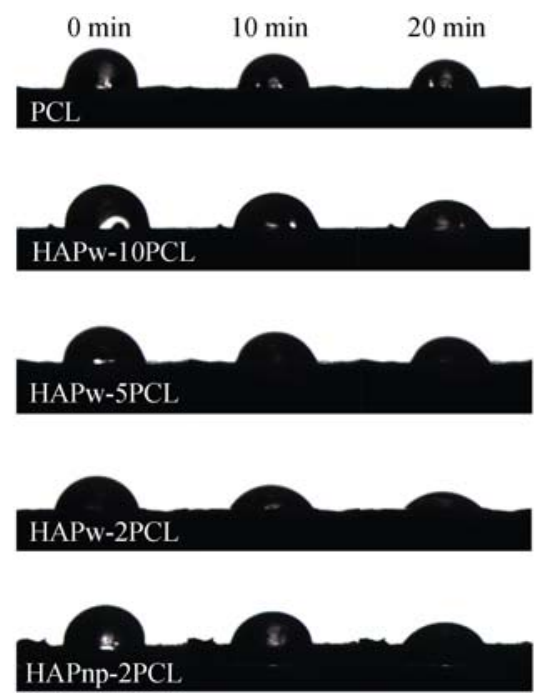

(B)

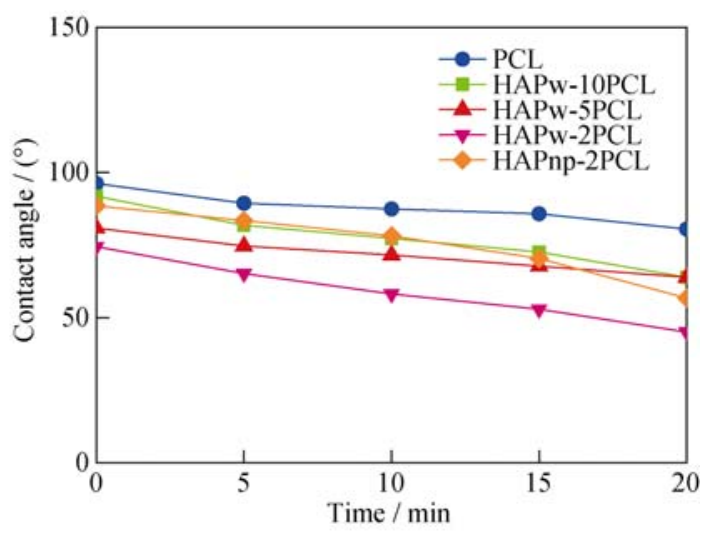

图 6 (A) PCL, HAPw-10PCL, HAPw-5PCL, HAPw-2PCL 和 HAPnp-2PCL 复合支架表面水滴在不同时间( $0 、 10 、 20$ min)的状态， (B)复合支架表面接触角室内空气下随时间的变化

Fig. 6 (A) Water contact angle of PCL scaffolds (first panel), HAPw-10PCL scaffolds (second panel), HAPw-5PCL scaffolds (third panel), HAPw-2PCL scaffolds (fourth panel), and HAPnp-2PCL. Ascending contact angles measured at varying times (0, 10, and 20 min), and (B) quantification of the contact angle with time 
可显著改善 PCL 材料的亲水性能。根据各支架 SEM 照片(图 3)显示, HAPw-PCL 复合支架表面的 PCL 膜层孔洞越大, 水滴渗透越快, 从而更快在支 架表面润湿; 而 HAPnp-2PCL 支架较为密实的 PCL 包裹表面则使其接触角与 HAPw-10PCL 支架相近。 已有研究报道说明支架表面亲/疏水特性是影响细 胞在支架上粘附增殖的重要因素之一, 与疏水支 架相比, 亲水性支架更有利于细胞播种、粘附和均 匀分布 ${ }^{[24]}$ 。

\section{$2.3 \mathrm{hBMSCs}$ 在 HAPw-PCL 复合支架上的增殖}

已有研究结果表明, 成骨细胞的增殖分化是骨 矿化发生前非常重要的阶段, 而成骨细胞增殖分化 的基本过程又受到细胞和材料相互作用的控制。本 研究考察了 hBMSCs 在各组复合支架表面上的增殖 分化情况 图 7 为 hBMSCs 在纯 PCL、HAPw-10PCL、 HAPw-5PCL 和 HAPw-2PCL 四组支架上培养 1、3 和 $7 \mathrm{~d}$ 后的细胞增殖情况。结果显示, 四组支架材料 都能够支持细胞增殖, 但是在 HAPw-PCL 复合支架 上的细胞增长率明显高于纯 $\mathrm{PCL}$ 支架 $(p<0.05)$, 随 着 $\mathrm{HAPw}$ 复合量的增加, hBMSCs 在支架表面的数 量也增加。

\section{$2.4 \mathrm{hBMSCs}$ 在 HAPw-PCL 复合支架上的碱 性磷酸酶(ALP)活性以及成骨基因的表达}

图 8 为 $\mathrm{hBMSCs}$ 在纯 PCL HAPw-10PCL HAPw5PCL 和 HAPw-2PCL 四组支架上培养 7 和 $14 \mathrm{~d}$ 后 的细胞 ALP 活性结果。四组支架的 ALP 活性结果 与细胞增殖结果类似, 培养 $14 \mathrm{~d}$ 后, HAPw-PCL 复 合支架比纯 PCL 支架表现出更高的 ALP 活性 $(p<$ $0.05)$, 并且随着 $\mathrm{HAPw}$ 复合量的增加, hBMSCs 的 ALP 活性也增加。

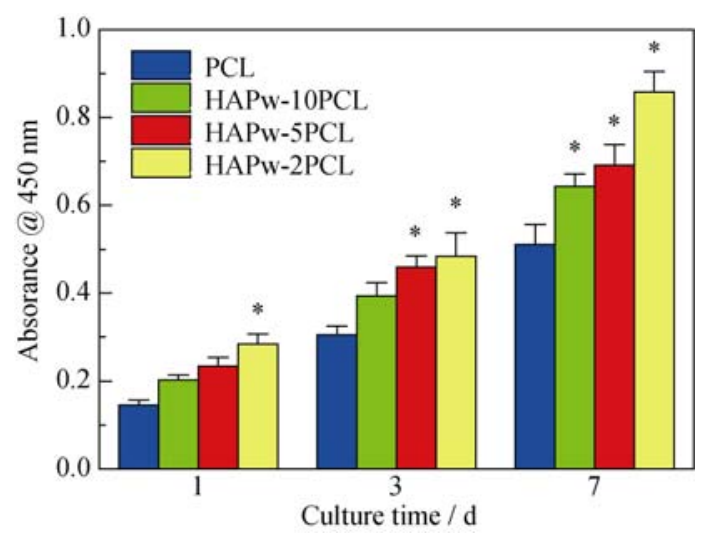

图 $7 \mathrm{hBMSCs}$ 在 PCL 和 HAPw-PCL 复合支架上培养 1、3、 $7 \mathrm{~d}$ 后的细胞增殖情况

Fig. 7 Proliferation of hBMSCs on the PCL and HAPw-PCL scaffolds for 1,3 and $7 \mathrm{~d}\left({ }^{*} p<0.05\right.$ if compared with the PCL scaffold control)

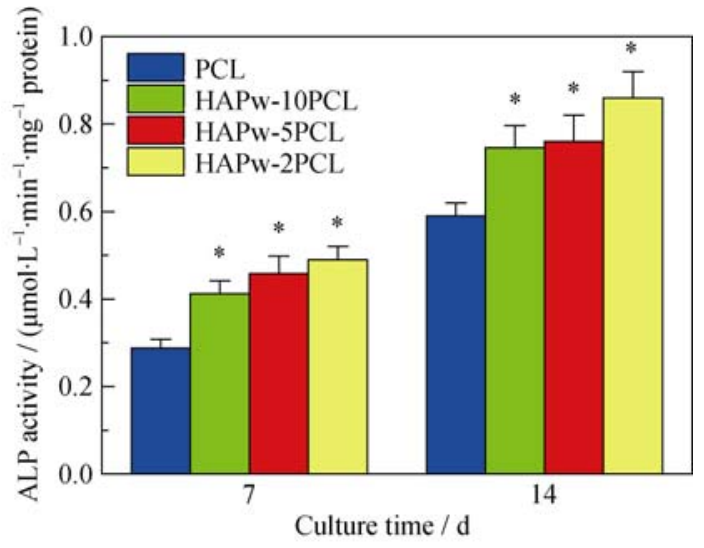

图 8 hBMSCs 在 PCL 和 HAPw-PCL 复合支架上培养 7、14 d 后的碱性磷酸酶活性结果

Fig. 8 ALP activity of hBMSCs cultured on PCL and HAPw-PCL scaffolds for 7 and $14 \mathrm{~d}\left({ }^{*} p<0.05\right.$ if compared with the PCL scaffold control)

图 9 为 $\mathrm{hBMSCs}$ 在 HAPw-PCL 复合支架上培养 7 和 $14 \mathrm{~d}$ 后的相关成骨基因表达(ALP, RUNX2, OCN), 由图可知, HAPw 的复合可以促进 hBMSCs 在 HAPw-PCL 复合支架上的成骨分化。HAPw-10PCL、 HAPw-5PCL 和 HAPw-2PCL 支架的 ALP、RUNX2、 OCN 基因表达明显高于纯 PCL 组支架 $(p<0.05)$, 说 明含 HAPw 的复合支架比单纯的 PCL 支架更好地 表达成骨特性。

已有研究结果表明, 在 PLA 支架中添加 HAP 不仅能够中和降解产物的酸性 ${ }^{[25]}$, 而且可以改善复 合支架的细胞粘附增殖 ${ }^{[26-27]}$ 。大量实验表明, HAP 不仅能够在成骨诱导剂存在的情况下显著促进成骨 细胞的成骨分化 ${ }^{[28-29]}$, 而且 HAP 与其他化学因子组 成的复合材料 ${ }^{[30-31]}$ 以及 HAP 所修饰的材料 ${ }^{[32-33]}$ 均 比不含 HAP 的对照组表现出更卓越的成骨诱导性 能, 甚至在无成骨诱导因子的情况下, 单纯的 HAP 也能表现出成骨诱导特性 ${ }^{[34]}$ 。另外, 有研究表明, 支架中孔的形状、大小和孔隙率直接影响着种植细 胞的迁移、分化和增殖 ${ }^{[35]}$ 。孔径太小则细胞的迁移 受到限制, 孔径过大则导致比表面积减小而限制细 胞的粘附和增殖, MC3T3-E1 细胞在孔径为 120 $325 \mu \mathrm{m}$ 的支架上的粘附、扩散和增殖相对较好 ${ }^{[36]}$ 。 对于 MC3T3-E1 细胞, 添加 $\mathrm{HAPw}$ 的 $\beta-\mathrm{TCP} / \mathrm{BG}$ 多孔 支架比纯 $\beta-\mathrm{TCP} / \mathrm{BG}$ 支架拥有更好的增殖效果 ${ }^{[37]}$ 。本 研究对 HAPw-PCL 复合支架的体外细胞相容性研 究发现, HAPw 能够改善 hBMSCs 的粘附、增殖和 分化。

\section{5 hBMSCs 在 HAPw-PCL 复合支架上的细} 胞外基质矿化

图 10 为 hBMSCs 在 HAPw-PCL 复合支架上培 

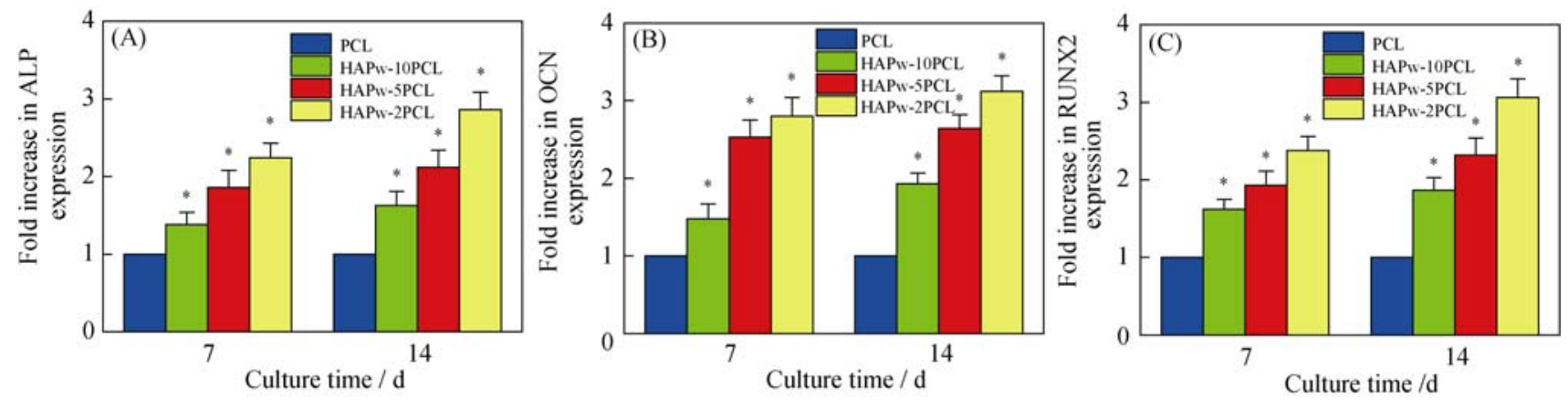

图 $9 \mathrm{hBMSCs}$ 在 HAPw-PCL 复合支架上培养 $7 \mathrm{~d}$ 和 $14 \mathrm{~d}$ 后的相关成骨基因表达(ALP(A), RUNX2(B), OCN(C))

Fig. 9 Osteogenic expression of ALP (A), OCN (B), RUNX2 (C) for hBMSCs cultured on the PCL and HAPw-PCL scaffolds by qRT-PCR analysis after $7 \mathrm{~d}$ and $14 \mathrm{~d}\left({ }^{*} p<0.05\right.$ if compared with the PCL scaffold control)

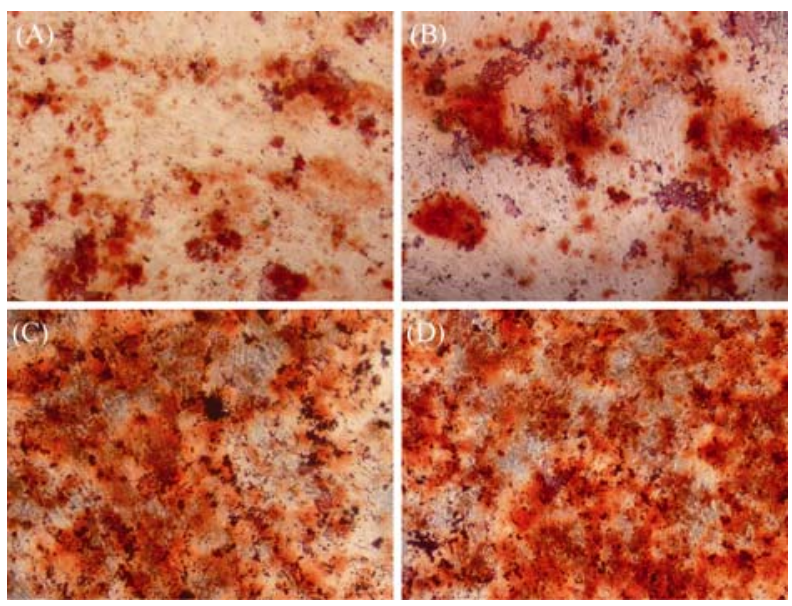

图 10 hBMSCs 在 PCL(A)、HAPw-10PCL (B)、HAPw-5PCL (C) 和 HAPw-2PCL (D)支架上培养 $21 \mathrm{~d}$ 后的 ECM 矿化情况 Fig. 10 ECM mineralization of hBMSCs on the PCL(A), HAPw-10PCL (B), HAPw-5PCL (C) and HAPw-2PCL (D) scaffolds after culturing for $21 \mathrm{~d}$

养 $21 \mathrm{~d}$ 后细胞外基质(ECM)的矿化情况。通过茜素 红染色可以看出, 细胞在四组支架上培养 $21 \mathrm{~d}$ 后都 有钙结节。相对于纯 PCL 组支架, HAPw-PCL 复合 支架上的钙结节明显增加, 表明 HAPw 能促进 hBMSCs 的细胞外基质矿化。

\section{3 结论}

运用三维打印技术成功制备了不同羟基磷灰石 晶须复合的 HAPw-PCL 支架，探究了复合支架的抗 压强度、生物活性及 hBMSCs 细胞在复合支架上的 成骨性能。结果表明, HAPw 含量增加导致 PCL 支架 强度显著提高至近 3 倍, 且支架表面由三维打印过 程中形成的 PCL 膜层随 HAPw 增多逐步缩小, 复合 支架表面亲水性则进一步增强。另外, HAPw 与 PCL 基材复合后, hBMSCs 细胞在支架内的粘附和铺展得 到改善, 由更高的 ALP 活性、成骨基因 OCN 和
RUNX2 表达以及细胞外基质矿化表明，细胞向成骨 方向分化能力也得到提升。因此，三维打印的 HAPw-PCL 支架表现出较好的骨修复应用潜力。

\section{参考文献:}

[1] CRANE G M, LSHAUG S L, MIKOS A G, et al. Bone Tissue Engineering. Nature Medicine, 1995, 1(12): 1322-1324.

[2] NAIR L S, LAURENCIN C T. Biodegradable polymers as biomaterials. Progress in Polymer Science, 2007, 32(8): 762-798.

[3] YUN H S, KIM S E, PARK E K. Bioactive glass-poly (E-caprolactone) composite scaffolds with 3 dimensionally hierarchical pore networks. Materials Science and Engineering C, 2011, 31(2): 198-205.

[4] ZHU N, LI M G, COOPER D, et al. Development of novel hybrid poly (L-lactide)/chitosan scaffolds using the rapid freeze prototyping technique. Biofabrication, 2011, 3(3): 390-395.

[5] ZHANG J H, ZHAO S C, ZHU Y F, et al. Three dimensional printing of strontium-containing mesoporous bioactive glass scaffolds for bone regeneration. Acta Biomaterialia, 2014, 10(5): 2269-2281.

[6] DOROZHKIN S V. Bioceramics of calcium orthophosphates. Biomaterials, 2010, 31(7): 1465-1485.

[7] SANTOS C, LUKLINSKA Z B, CLARKE R L, et al. Hydroxyapatite as a filler for dental composite materials: mechanical properties and in vitro bioactivity of composites. Journal of Materials Science: Materials in Medicine, 2001, 12(7): 565-573.

[8] ZHANG H, DARVELL B W. Mechanical properties of hydroxyapatite whisker-reinforced bis-GMA-based resin composites. Dental Materials Official Publication of the Academy of Dental Materials, 2012, 28(8): 824-830.

[9] GAO W M, RUAN C X, CHEN Y F. Effects of hydroxyapatite morphology on the mechanical strength of hydroxyapatite- polyanhydride composites. Journal of Materials Science \& Engineering, 2006, 24(5): 636-646.

[10] KANE R J, CONVERSE G L, ROEDER R K. Effects of the reinforcement morphology on the fatigue properties of hydroxyapatite reinforced polymers. Journal of the Mechanical Behavior of Biomedical Materials, 2008, 1(3): 261-268.

[11] ZHANG H Q, DARVELL B W. Failure and behavior in water of hydroxyapatite whisker-reinforced bis-GMA-based resin composites. Journal of the Mechanical Behavior of Biomedical Materials, 2012, 10(6): 39-47.

[12] CONVERSE G L, YUE W, ROEDER R K. Processing and tensile properties of hydroxyapatite whisker reinforced polyetheretherketone. Biomaterials, 2007, 28(6): 927-935.

[13] $\mathrm{HU} \mathrm{H}, \mathrm{XU} \mathrm{G}, \mathrm{ZAN} \mathrm{Q}$, et al. In situ formation of nano hydroxyapa- 
tite whisker reinforced porous $\beta$-TCP scaffolds. Microelectronic Engineering, 2012, 98: 566-569.

[14] JANSEN E J, SLADEK R E, BAHAR H, et al. Hydrophobicity as a design criterion for polymer scaffolds in bone tissue engineering. Biomaterials, 2005, 26(21): 4423-4431.

[15] WHANG K, GOLDSTICK T K, HEALY K E. A biodegradable polymer scaffold for delivery of osteotropic factors. Biomaterials, 2000, 21(24): 2545-2551.

[16] WU C, ZHOU Y, XU M, et al. Copper-containing mesoporous bioactive glass scaffolds with multifunctional properties of angiogenesis capacity, osteostimulation and antibacterial activity. Biomaterials, 2013, 34(2): 422-433.

[17] PARK S A, LEE S H, KIM W D. Fabrication of porous polycaprolactone/hydroxyapatite (PCL/HA) blend scaffolds using a 3D plotting system for bone tissue engineering. Bioprocess and Biosystems Engineering, 2011, 34(4): 505-513.

[18] SHI G Q. Individualized artificial knee joint design and manufacturing based on CAD software. Journal of University of Shanghai for Science and Technology, 2013, 35(1): 82-86.

[19] CIOCCA L, DE CF, FANTINI M, et al. CAD/CAM and rapid prototyped scaffold construction for bone regenerative medicine and surgical transfer of virtual planning: a pilot study. Computerized Medical Imaging \& Graphics, 2009, 33(1): 58-62.

[20] MATSUBARA T, SUARDITA K, ISHII M, et al. Alveolar bone marrow as a cell source for regenerative medicine: differences between alveolar and iliac bone marrow stromal cells. Journal of Bone and Mineral Research, 2005, 20(3): 399-409.

[21] ASSA S, LARON Z. Preparation and characterization of dense nanohydroxyapatite/PLLA composites. Materials Science and Engineering C, 2009, 29(1): 172-177.

[22] HAYATI A N, HOSSEINALIPOUR S M, REZAIE H R, et al. Characterization of poly(3-hydroxybutyrate)/nano-hydroxyapatite composite scaffolds fabricated without the use of organic solvents for bone tissue engineering applications. Materials Science \& Engineering C, 2012, 32(3): 416-422.

[23] ZHANG R Y, MA P X. Poly( $\alpha$-hydroxyl acids)/hydroxyapatite porous composites for bone-tissue engineering. I. Preparation and morphology. Journal of Biomedical Materials Research Part A, 1999, 44(4): 446-455.

[24] FLEISCHER S, SHAPIRA A, REGEV O, et al. Albumin Fiber Scaffolds for Engineering Functional Cardiac Tissues. Biotechnology and Bioengineering, 2014, 11(6): 1246-1257.

[25] HUTMACHER D W. Scaffolds in tissue engineering bone and cartilage. Biomaterials, 2001, 21(24): 2529-2543.
[26] BOMBONATO-PRADO K, BELLESINI L C, MARQUES M, et al. Microarray-based gene expression analysis of human osteoblasts in response to different biomaterials. Journal of Biomedical Materials Research Part A, 2009, 88(2): 401-408.

[27] SOHN S H, JUN H K, KIM C S, et al. Biological responses in osteoblast-like cell line according to thin layer hydroxyapatite coatings on anodized titanium. Journal of Oral Rehabilitation, 2006, 33(12): 898-911.

[28] HUANG Y C, HSIAO P C, CHAI H J. Hydroxyapatite extracted from fish scale: effects on MG63 osteo6last-like cells. Ceramics International, 2011, 37(6): 1825-1831.

[29] LIU Y K, WANG G C, CAI Y R, et al. In vitro effects of nanophase hydroxyapatite particles on proliferation and osteogenic differentiation of bone marrow-derived mesenchymal stem cells. Journal of Biomedical Materials Research Part A. 2009, 90(4): 1083-1091.

[30] BABISTER J C, HAILS L A, OREFFO R O, et al. The effect of pre-coating human bone marrow stromal cells with hydroxyapatite/ amino acid nanoconjugates on osteogenesis. Biomaterials, 2009, 30(18): 3174-3182.

[31] LUO E, LIU X, WEI S C, et al. Osteoblast adhesion to clodronatehydroxyapatite composite. Applied Surface Science, 2008, 255(2): 308-311.

[32] DIMITRIEVSKA S, BUREAU MN, ANTONIOU J, et al. Titanichydroxyapatite nanocomposite coatings support human mesenchymal stem cells osteogenic differentiation. Journal of Biomedical Materials Research Part A, 2011, 98(4): 576-588.

[33] ZHAO H, DONG W, ZHENG Y, et al. The structural and biological properties of hydroxyapatite-modified titanate nanowire scaffolds. Biomaterials, 2011, 32(25): 5837-5846.

[34] HU Q H, TAN Z, LIU Y K, et al. Effect of crystallinity of calcium phosphate nanoparticles on adhesion proliferation, and differentiation of bone marrow mesenchymal stem cells. Journal of Materials Chemistry, 2007, 17(44): 4690-4698.

[35] LI Y, MA T, YANG S T, et al. Thermal compression and characterization of three-dimensional nonwoven PET matrices as tissue engineering scaffolds. Biomaterials, 2001, 22(6): 609-618.

[36] MURPHY C M, HAUGH M G, O'BRIEN F J. The effect of mean pore size on cell attachment, proliferation and migration in collagen glycosaminoglycan scaffolds for bone tissue engineering. Biomaterials, 2010, 31(3): 461-466.

[37] HU H T, XU G H, ZAN Q F. In situ formation of nano-hydroxyapatite whisker reinfoced porous b-TCP scaffolds. Microelectronic Engineering, 2012, 98: 566-569. 